\title{
Extending the interval for port-a-cath maintenance
}

\author{
Eugenia Girda, Rebecca Phaeton, Gary L. Goldberg, Dennis Kuo*
}

Department of Obstetrics \& Gynecology and Women's Health, Division of Gynecologic Oncology, Albert Einstein College of Medicine and Montefiore Medical Center, New York City, USA; ${ }^{*}$ Corresponding Author: DYKUO@,montefiore.org

Received 18 February 2013; revised 20 March 2013; accepted 28 March 2013

Copyright (C) 2013 Eugenia Girda et al. This is an open access article distributed under the Creative Commons Attribution License, which permits unrestricted use, distribution, and reproduction in any medium, provided the original work is properly cited.

\section{ABSTRACT}

Objective: The objective of this study is to assess the outcome of port-a-cath (PAC) maintenance every three months in patients with gynecologic malignancies with the goal of standardizing a safe and appropriate interval that would maintain patency and minimize side effects. Methods: We performed a retrospective medical record review and evaluation of all patients with a Bard $^{\oplus}$ PAC who were noted to have no evidence of disease (NED) during the years 2003 to 2010. The interval between accessions and any complications related to the presence of the PAC were recorded. Relevant complications included skin infections, bacteremia, thrombosis, and occlusions. Statistical analysis was done using the Fisher's exact test. Results: A total of 201 patients had PAC placed and 43 patients underwent PAC accessions to maintain patency. The total number of accessions was 150 with a median number per patient of 2.0 (range 1 - 10). The mean time between flushes was 112 days $(S D=57)$. When comparing women in maintenance who had flushes within 90 days versus those who had flushes over 90 days apart, there was no difference in infection or occlusion rates between these groups $(p=$ $0.515)$. In the <90-day group, one patient was noted to have resistance during the flush. In the $\geq 90$-day group, one patient had an occlusion that did not resolve with Alteplase. Another patient had skin erythema with spontaneous resolution. Conclusion: Infections and occlusions are rare in women with gynecologic malignancies undergoing maintenance of their PAC. Longer intervals between PAC flushes do not appear to affect the outcome in our patients. Our ongoing data and follow-up confirm that extending the interval of PAC accession to every 3 months, rather than monthly, is safe, effective and con- venient in the patient population with gynecologic malignancies.

Keywords: Indwelling Port-A-Cath; Flushing; Complication; Prevention

\section{INTRODUCTION}

The safe and timely administration of chemotherapy in part relies on consistent access to the venous system. In many patients with advanced malignancies and other medical co-morbidities, such as peripheral vascular disease, a more reliable form of access is mediated by semi-permanent or permanent devices such as a peripherally inserted central catheter (PICC), Hickman catheter, or port-a-cath (PAC). Implantable venous access devices provide repeated access to the vascular system and have been widely used in patients with malignancies receiving long-term treatments such as chemotherapy and total parenteral nutrition (TPN).

Port-A-Cath consists of a port and an intravascular device. The intravascular device is inserted into a subclavian or jugular vein and tunneled under the skin of the chest wall. The procedure is usually performed under sedation or general anesthetic. As the breach of skin integrity does not exceed the caliber of the needle, the risk of infection and thrombosis is lower compared to indwelling catheters such as Hickman catheters [1]. Many studies have analyzed the complications associated with implantable catheters, however very few have commented on the ideal or recommended length of the maintenance period between flushing the port with heparin [2-5].

At our institution, several patients, at the completion of the chemotherapy course, retain their PAC due to the high recurrence rate of some of these malignancies and therefore a possible need for subsequent use of PAC for additional chemotherapy cycles after advisement by their provider. During this maintenance period, many of our patients are no longer receiving chemotherapy or having regular blood tests performed and have relatively poor 
compliance with their regular port accession appointments. At present, the ideal interval of accession to maintain patency is unknown. Therefore, it is important to develop a safe, cost effective and convenient catheter maintenance protocol.

Although the manufacturer's guidelines usually recommend monthly accession in order to maintain port patency, based on our previous data and safety profile we currently access the PAC every three months to maintain port patency [6]. The purpose of this study is to assess the follow up and outcome of PAC maintenance every 3 months in patients with gynecologic cancers and provide a more comprehensive analysis of longer-term data.

\section{METHODS}

All patients who underwent care in the Gynecologic Oncology Division of the Department of Obstetrics and Gynecology and Women's Health at the Albert Einstein College of Medicine and Montefiore Medical Center were considered for entry into the study. The placement of PAC and subsequent follow-up was derived from both a computer database and complementary medical records at the divisions' administrative offices. The computer database and the medical records were accessed to obtain a listing of all patients who had a procedure of PAC insertion. Review and screening of all the charts was performed to include eligible patients.

Inclusion criteria were: confirmed placement of PAC on imaging, subsequent treatment with the practice as well as follow up during the maintenance period. The exclusion criteria were: patients who were receiving anticoagulation with Lovenox, Fragmin, or Coumadin for other medical issues or those who had PAC device removed immediately after first-line chemotherapy.

Maintenance period was defined as a minimum of six months without chemotherapy, intravenous hydration or other infusion. The protocol for PAC access used standard sterile precautions using $10 \mathrm{cc}$ of normal saline followed by $5 \mathrm{cc}$ of heparin flush (100 units heparin/cc). If there was no blood return, Alteplase was administered and results noted.

Chart review included collection of data such as age, date of PAC insertion, location of PAC and dates of PAC accessions. Complications associated with PAC flushing, including catheter tip thrombus and infections, were recorded as well as results of managing these complications. The data was subsequently entered into an Excel spreadsheet. From the collected data, the total number of port access flushes and the interval in days between each flush was calculated. Statistical analysis was performed using the student $t$ test.

\section{RESULTS}

A total of 201 patients had a PAC placed between
2003 and 2010 at Montefiore Medical Center. Twentyone percent (43) of patients retained their port at the completion of chemotherapy and had continued PAC accessions to maintain patency. Of the patients who were excluded (158), one hundred and six (106) patients did not have adequate maintenance period due to either hospitalization or continued chemotherapy, thirty nine (39) patients died following PAC placement, eight (8) patients had PAC removed following chemotherapy and five (5) patients transferred care or were lost to follow-up.

Forty-three patients who were included in the study had been scheduled for PAC maintenance every 3 months. The primary malignancies for these patients were as follows: 25 patients with uterine cancers, 15 patients with ovarian cancers, and 3 patients with cervical cancer (Table 1).

Compliance with visits for PAC maintenance varied significantly with the individual mean accession times varying between 30 days and 210 days. The total number of accessions was 150 with a median number per patient of 2.0 (range 1 - 10). The mean time between flushes was 112 days $(\mathrm{SD}=57)$. Figure 1 shows the distribution of mean accession intervals for all of the patients.

Since our initial pilot study analyzed a 90-day maintenance follow-up, we aimed to determine if extending maintenance beyond 90 days was associated with any difference in side effects. Therefore, the accession intervals were divided into two groups: greater than 90 days and fewer than 90 days. The interval of accessions, right before each of the complications, was recorded. In the group with less than 90-day intervals, 30 accessions were noted and 1 complication was observed. In the second group with flush intervals more than 90 days apart, 111 accessions and 2 complications were noted.

There was no statistical difference in infection or occlusion rates between these groups $(p=0.515)$. Table 2 notes the complications associated with PAC accessions. In the <90-day group, one patient had resistance with slow return of blood during flushing. This patient had two port accessions with the interval of 30 days, and the complication was noted at the time of second port accession. In the $\geq 90$-day group, one patient had an occlusion that did not respond to Alteplase. This patient had two PAC accessions, which were 92 days apart. Another patient in the $\geq 90$-day group had skin erythema around the PAC flush site. This patient has 11 port accessions for

Table 1. Types of malignancies.

\begin{tabular}{cc}
\hline Type of Tumor & No. of patients \\
\hline Uterine & 25 \\
Ovarian & 15 \\
Cervical & 3 \\
\hline
\end{tabular}




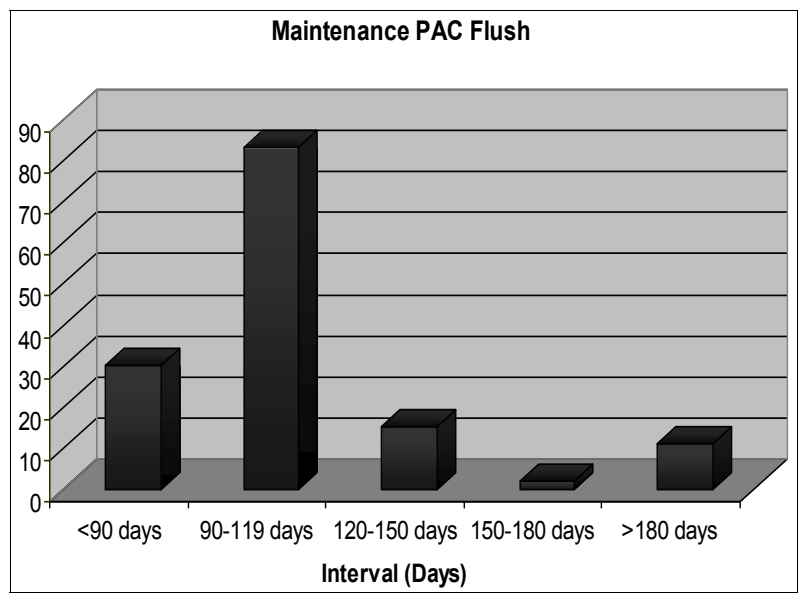

Figure 1. Distribution of mean accession intervals.

Table 2. Complications associated with PAC accessions.

\begin{tabular}{ccccc}
\hline $\begin{array}{c}\text { Patient } \\
\text { No. }\end{array}$ & $\begin{array}{c}\text { Date of Last } \\
\text { Accession }\end{array}$ & $\begin{array}{c}\text { Date of } \\
\text { Complication }\end{array}$ & $\begin{array}{c}\text { No. of Days } \\
\text { between }\end{array}$ & Complication \\
\hline 1 & $4 / 16 / 2010$ & $7 / 30 / 2010$ & 105 & Erythema \\
2 & $5 / 1 / 2008$ & $5 / 31 / 2008$ & 30 & Resistance \\
3 & $8 / 20 / 2010$ & $11 / 20 / 2010$ & 92 & Occlusion \\
\hline
\end{tabular}

patency with intervals ranging between 91 and 338 days.

The complication occurred at the eleventh PAC flush with preceding interval of 105 days. There were no other complications, such as fever, DVT, or infection noted during the time of remaining maintenance accessions. It is important to note that 10 patients had greater than 5 maintenance periods in the $\geq 90$-day group without any complications, which may suggest that prolonged maintenance periods of PAC is not a factor in causing complications, such as thrombosis of the catheter.

\section{DISCUSSION}

To date, there has been no standardized assessment of the safe and appropriate interval between maintenance flushing of an indwelling PAC. As gynecologic oncologists who administer chemotherapy, we have a unique perspective about long-term complications of PAC placement as we are both the surgeons who insert them and manage potential acute complications such as pneumothorax, infection, bleeding, pain, and determine the interval of follow up, as well as schedule and perform removal of these devices. As such, we have a responsibility beyond the manufacturer's label to ensure that the suggestions have scientific merit, are safe for our patients, and should be adopted into daily practice.

Our ongoing data has shown that in women with gynecologic malignancies undergoing every 90-day PAC accessions to maintain patency, infections and occlusions are rare. Our extended results are consistent with our previous pilot study demonstrating that longer intervals between PAC flushes do not increase the catheter complication rate.

This retrospective analysis of a relatively small population limited to one geographic location has certain limitations. In our initial pilot study, 7 out of 73 patients had no blood return during PAC accession. When analyzing average interval between accessions, no statistical significant was noted between these patients compared to those patients without any difficulty with port accessions [6]. Our initial and now follow-up data suggest that extending the interval of PAC accession to every 3 months, rather than monthly, is safe, effective and convenient in maintaining PAC patency in this patient population. Furthermore, more frequent flushing can reach the limit of maximum accessions of a PAC device if the maintenance period is prolonged in cases where malignancy does not recur. Accessing the device too frequently can potentially cause more residual blood in the catheter and further limit the successful blood return during the flushing.

Furthermore, catheter maintenance is associated with significant costs, which primarily affect the patients and the healthcare system. Although cost was not considered in this analysis, it can be assumed that more frequent flushing is more costly and burdensome not only to the medical system, but for patient transportation and compliance. Indeed, for women who have completed their therapy, monthly visits to the doctor's office for PAC flush have resulted in poor patient compliance, as it is invasive, inconvenient and expensive. Thus, extending the interval for PAC maintenance period is feasible, safe, convenient and cost effective. It will facilitate patient's follow up and compliance. A three-month catheter maintenance schedule would also coincide with patient's required clinical cancer follow up office visits and may improve their compliance and satisfaction, while decreasing healthcare costs.

\section{REFERENCES}

[1] Ng, F., Mastoroudes, H., Paul, E., Davies, N., Tibballs, J., Hochhauser, D., Mayer, A., Begent, R. and Meyer, T. (2007) A comparison of Hickman line-and port-a-cath-associated complications in patients with solid tumours undergoing chemotherapy. Clinical Oncology, 7, 551-556. doi:10.1016/j.clon.2007.04.003

[2] Sanchez, Y.L., Caravajal, J.M., Fuster, D. and Eiras, M.E. (2006) Protocol for the implantation of a venous access device (port-a-cath system). The complications and solutions found in 560 cases. Clinical and Translational Oncology, 10, 735-741. doi:10.1007/s12094-006-0120-y

[3] Coccaro, M., Bochicchio, A.M., Capobianco, A.M., Di Leo, P., Mancino, G. and Cammarota, A. (2001) Longterm infusional systems: Complications in cancer patients. Tumori, 87, 308-311.

[4] Vandoni, R.E., Guerra, A., Sanna, P., Bogen, M., Cavalli, 
F. and Gertsch, P. (2009) Randomized comparison of complications from three different permanent central venous access systems. Swiss Medical Weekly, 139, 313316.

[5] Kock, H.J., Pietsch, M., Krause, U., Wilke, H. and Eigler, F.W. (1998) Implantable vascular access systems: Experience in 1500 patients with totally implanted central venous port systems. World Journal of Surgery, 22, 1216. doi: $10.1007 / \mathrm{s} 002689900342$

[6] Kuo, Y.S., Schwartz, B., Santiago, J. and Anderson, P.S. (2005) How often should a port-a-cath be flushed? Cancer Investigation, 23, 582-585. doi: $10.1080 / 07357900500276923$ 